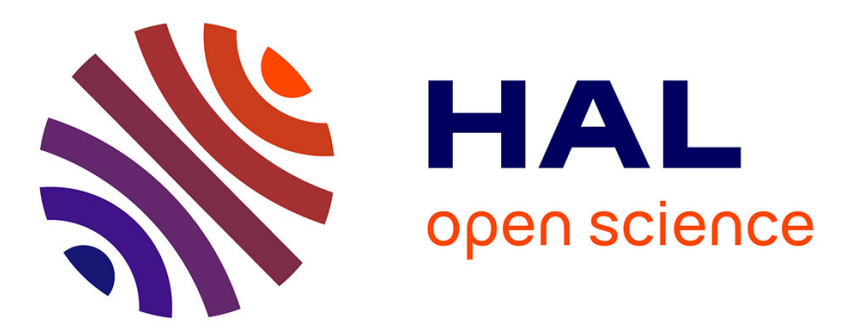

\title{
Perturbation and sensitivity calculations for time eigenvalues using the Generalized Iterated Fission Probability
}

\author{
A. Jinaphanh, A. Zoia
}

\section{- To cite this version:}

A. Jinaphanh, A. Zoia. Perturbation and sensitivity calculations for time eigenvalues using the Generalized Iterated Fission Probability. Annals of Nuclear Energy, 2019, 133, pp.678 - 687. 10.1016/j.anucene.2019.06.062 . hal-03487336

\section{HAL Id: hal-03487336 https://hal.science/hal-03487336}

Submitted on 20 Dec 2021

HAL is a multi-disciplinary open access archive for the deposit and dissemination of scientific research documents, whether they are published or not. The documents may come from teaching and research institutions in France or abroad, or from public or private research centers.
L'archive ouverte pluridisciplinaire HAL, est destinée au dépôt et à la diffusion de documents scientifiques de niveau recherche, publiés ou non, émanant des établissements d'enseignement et de recherche français ou étrangers, des laboratoires publics ou privés.

\section{(ㄷ)(1) $\$$}

Distributed under a Creative Commons Attribution - NonCommercial| 4.0 International 


\title{
Perturbation and sensitivity calculations for time eigenvalues using the Generalized Iterated Fission Probability
}

\author{
A. Jinaphanh ${ }^{\mathrm{a}, *}$, A. Zoia ${ }^{\mathrm{a}}$ \\ ${ }^{a}$ DEN-Service d'études des réacteurs et de mathématiques appliquées (SERMA), CEA, Université \\ Paris-Saclay, F-91191, Gif-sur-Yvette, France
}

\begin{abstract}
Time eigenvalues emerge in several key applications related to neutron transport problems, including reactor start-up and reactivity measurements. In this context, experimental validation and uncertainty quantification would demand to assess the variation of the dominant time eigenvalue in response to a variation of nuclear data. Attention has been recently drawn to the development of theoretical and numerical tools for a first-order Standard Perturbation Theory (SPT) applied to time eigenvalues, which can be thought of as an extension of the well-known SPT formalism for $k$-eigenvalues. In this work we present a new Monte Carlo method enabling first-order perturbation and sensitivity analysis for time eigenvalues, based on the Generalized Iterated Fission Probability approach that we have recently introduced. The proposed method is detailed and its accuracy is assessed by considering a few relevant verification tests.
\end{abstract}

Keywords: Monte Carlo, Time eigenvalues, Criticality, Sensitivity, Perturbation, IFP

\footnotetext{
*Corresponding author

Email addresses: alexis.jinaphanh@cea.fr (A. Jinaphanh), andrea.zoia@cea.fr (A. Zoia)

Preprint submitted to Elsevier

June 28, 2019
} 


\section{Introduction}

The rediscovery of the Iterated Fission Probability (IFP) method has made possible to compute first-order reactivity perturbations and sensitivity coefficients for $k$ eigenvalue calculations by continuous-energy Monte Carlo methods (Nauchi and Kameyama, 2010; Kiedrowski et al., 2011), thus enabling the implementation of Standard Perturbation Theory (SPT) estimators in production Monte Carlo codes (Terranova et al., 2018; Choi et al., 2018; Kiedrowski and Brown, 2013; Jinaphanh et al., 2016; Aufiero et al., 2015; Perfetti et al., 2016; Qiu et al., 2016); for a comprehensive review, see, e.g., (Kiedrowski, 2017).

The $k$ eigenvalues are intrinsically related to the behaviour of neutron generations (Bell and Glasstone, 1970). In many technological applications, encompassing reactor start-up (Pfeiffer et al., 1974) and pulsed neutron reactivity measurements (Cao and Lee, 2010), one is led to explicitly consider the evolution of the neutron population with respect to time instead of generations: this is tantamount to examining the $\alpha$ eigenvalues, which are related to the asymptotic reactor period and to the prompt neutron decay constant (Bell and Glasstone, 1970). In this context, experimental validation, uncertainty quantification and bias estimation through data assimilation would demand the perturbation effect and/or sensitivity coefficients of the dominant $\alpha$-eigenvalue of the neutron flux with respect to variation in nuclear data.

Perturbation and sensitivity analysis of $\alpha$ eigenvalues has recently attracted much attention, and several attempts have been made at extending the first-order SPT methods for both deterministic (Endo and Yamamoto, 2018; Favorite, 2018) and Monte Carlo solvers (Yamamoto and Sakamoto, 2019; Burke et al., 2019; Jinaphanh and Zoia, 2019). Based on the Generalized IFP method proposed in (Terranova et al., 2017), which can estimate the adjoint fundamental mode as- 
sociated to the $\alpha$-eigenvalue equation, and more generally ratios of bilinear forms involving the direct and adjoint fundamental $\alpha$-modes, we have implemented a new family of Monte Carlo estimators for perturbations and sensitivities coefficients of the $\alpha$ eigenvalue with respect to nuclear data (Jinaphanh and Zoia, 2019). This method was initially verified for simplified multi-group neutron transport problems. In this work we extend our findings by considering full continuousenergy transport: for this purpose, the algorithm sketched in (Jinaphanh and Zoia, 2019) has been implemented in the Monte Carlo code TRIPOLI- $4{ }^{\circledR}$, developed at CEA (Brun et al., 2015).

This paper is structured as follows: in Sec. 2 we will recall the Monte Carlo implementation of direct and adjoint $\alpha$-eigenvalue calculations and the Generalized IFP method. Then, in Sec. 3 we will illustrate the formula for the first-order perturbations of the $\alpha$ eigenvalue (including the contributions due to delayed neutrons) and show how these quantities can be computed by the Generalized IFP method, once appropriate estimators are introduced; these results will be extended to the sensitivity analysis in Sec. 4. In Sec. 5 the proposed methods will be verified against exact solutions for a few relevant benchmark configurations; furthermore, the continuous-energy implementation of TRIPOLI- $4{ }^{\circledR}$ will be contrasted to the results of the deterministic solver developed in (Endo and Yamamoto, 2018) for the HEU-SOL-THERM-012 benchmark. Conclusions will be finally drawn in Sec. 6. 


\section{Monte Carlo methods for direct and adjoint $\alpha$-eigenvalue problems}

The time-dependent neutron and precursor behaviour in a multiplying system is described by the following system of equations

$$
\begin{aligned}
& \frac{1}{v} \frac{\partial}{\partial t} \varphi(\mathbf{r}, \mathbf{\Omega}, E, t)+\mathcal{L} \varphi(\mathbf{r}, \mathbf{\Omega}, E, t)=\mathcal{F}_{p} \varphi(\mathbf{r}, \mathbf{\Omega}, E, t)+\sum_{j} \frac{\chi_{d, j}(\mathbf{r}, E)}{4 \pi} \lambda_{j} c_{j}(\mathbf{r}, t)+Q \\
& \frac{\partial}{\partial t} c_{j}(\mathbf{r}, t)=\iint v_{d, j}\left(E^{\prime}\right) \Sigma_{f}\left(\mathbf{r}, E^{\prime}\right) \varphi\left(\mathbf{r}, \mathbf{\Omega}^{\prime}, E^{\prime}, t\right) d \mathbf{\Omega} d E-\lambda_{j} c_{j}(\mathbf{r}, t),
\end{aligned}
$$

where the Boltzmann equation (1) for the neutron flux $\varphi(\mathbf{r}, \boldsymbol{\Omega}, E, t)$ at position $\mathbf{r}$, direction $\boldsymbol{\Omega}$, energy $E$ and time $t$ is coupled to the evolution equations (2) for the precursor concentration $c_{j}(\mathbf{r}, t)$ of family $j$ (Bell and Glasstone, 1970). Here $\mathcal{L}$ denotes the net disappearance operator,

$\mathcal{L} \varphi(\mathbf{r}, \mathbf{\Omega}, E, t)=\mathbf{\Omega} \cdot \nabla \varphi+\Sigma_{t} \varphi-\iint \Sigma_{s}\left(\mathbf{r}, \mathbf{\Omega}^{\prime}, E^{\prime} \rightarrow \mathbf{\Omega}, E\right) \varphi\left(\mathbf{r}, \mathbf{\Omega}^{\prime}, E^{\prime}, t\right) d \mathbf{\Omega}^{\prime} d E^{\prime}$

and $\mathcal{F}_{p}$ the prompt fission operator,

$$
\mathcal{F}_{p} \varphi(\mathbf{r}, \mathbf{\Omega}, E, t)=\frac{\chi_{p}(\mathbf{r}, E)}{4 \pi} \iint v_{p}\left(E^{\prime}\right) \Sigma_{f}\left(\mathbf{r}, E^{\prime}\right) \varphi\left(\mathbf{r}, \mathbf{\Omega}^{\prime}, E^{\prime}, t\right) d \mathbf{\Omega}^{\prime} d E^{\prime} .
$$

Notation is as follows: $\Sigma_{t}(\mathbf{r}, E)$ denotes the total cross section, $\Sigma_{s}\left(\mathbf{r}, \mathbf{\Omega}^{\prime}, E^{\prime} \rightarrow\right.$ $\mathbf{\Omega}, E)$ the scattering transfer kernel and $\Sigma_{f}(\mathbf{r}, E)$ the fission cross section; $v_{p}(E)$ denotes the average number of prompt fission neutrons and $v_{d, j}(E)$ average number of delayed fission neutrons for precursor family $j ; \chi_{p}(\mathbf{r}, E)$ denotes the prompt fission spectrum and $\chi_{d, j}(\mathbf{r}, E)$ the delayed fission spectrum for precursor family $j ; \lambda_{j}$ denote the delayed neutron precursor decay constants for family $j$. In order to keep notation to a minimum, we have considered a single fissile nucleus: the generalization to several nuclei is straightforward. Finally, the term $Q$ represents 
the external neutron source. The kinetic equations must be assigned proper initial and boundary conditions for $\varphi(\mathbf{r}, \boldsymbol{\Omega}, E, t)$ and $c_{j}(\mathbf{r}, t)$. All physical parameters have been supposed time-independent.

Time eigenvalue equations (often called $\alpha$-eigenvalue equations) are obtained from Eqs. (3) and (4) by postulating variable separation and assuming a timedependence of the kind $\exp (\alpha t)$ for the flux and the precursors, for a given set of eigenvalues $\alpha$ and time-independent eigenfunctions $\varphi_{\alpha}(\mathbf{r}, \boldsymbol{\Omega}, E)$ and $c_{\alpha, j}(\mathbf{r})($ Bell and Glasstone, 1970). Plugging the eigenvalue equations for the precursors into that for the neutrons yields

$$
\frac{\alpha}{v} \varphi_{\alpha}(\mathbf{r}, \boldsymbol{\Omega}, E)+\mathcal{L} \varphi_{\alpha}(\mathbf{r}, \boldsymbol{\Omega}, E)=\mathcal{F}_{p} \varphi_{\alpha}(\mathbf{r}, \boldsymbol{\Omega}, E)+\sum_{j} \frac{\lambda_{j}}{\lambda_{j}+\alpha} \mathcal{F}_{d, j} \varphi_{\alpha}(\mathbf{r}, \mathbf{\Omega}, E),
$$

where we have introduced the delayed fission operator

$$
\mathcal{F}_{d, j} \varphi_{\alpha}(\mathbf{r}, \mathbf{\Omega}, E)=\frac{\chi_{d, j}(\mathbf{r}, E)}{4 \pi} \iint v_{d, j}\left(E^{\prime}\right) \Sigma_{f}\left(\mathbf{r}, E^{\prime}\right) \varphi_{\alpha}\left(\mathbf{r}, \mathbf{\Omega}^{\prime}, E^{\prime}\right) d \mathbf{\Omega}^{\prime} d E^{\prime}
$$

The fundamental eigen-pair $\left\{\alpha, \varphi_{\alpha}\right\}$ of Eq. (5) can be determined by Monte Carlo methods by using the $\alpha-k$ power iteration, originally proposed for prompt decay constants (Brockway et al., 1985) and later extended to the general case with neutrons and precursors (Zoia et al., 2015). In order for this paper to be selfcontained, we briefly recall the algorithm here: for more details, the reader is referred to (Zoia et al., 2015). The basic idea is to add a factor $1 / k$ that multiplies the production terms and to iteratively adjust the $\alpha$ value such that Eq. (5) is satisfied with $k=1$. For positive $\alpha$, the term $\Sigma_{\alpha}=\alpha / v$ on the left hand side is interpreted as a "capture" cross section in the modified power iteration (Brockway et al., 1985; Cullen, 2003). For negative $\alpha, \Sigma_{\alpha}$ is moved to the right hand side of the equation and interpreted as a "production" term (Brockway et al., 1985; Cullen, 2003). The standard implementation of this algorithm 
has been shown to be numerically unstable for negative $\alpha$, possibly leading to abnormal termination (Hill, 1983). An improved algorithm that overcomes these limitations has been proposed (Zoia et al., 2014, 2015; Mancusi and Zoia, 2018): Eq. (5) is formally rearranged in order to preserve the balance between destructions and productions, namely,

$$
\mathcal{L} \varphi_{\alpha}+\Sigma_{\alpha, \eta} \varphi_{\alpha}=\mathcal{F}_{p} \varphi_{\alpha}+\sum_{j} \frac{\lambda_{j}}{\lambda_{j}+\alpha} \mathcal{F}_{d, j} \varphi_{\alpha}+\mathcal{F}_{\alpha, \eta} \varphi_{\alpha}
$$

where we have introduced a positive $\alpha$-absorption cross section $\Sigma_{\alpha, \eta}=-\eta \alpha / v>0$, $\eta$ being an arbitrary positive constant, and the associated $\alpha$-production operator

$$
\mathcal{F}_{\alpha, \eta} \varphi_{\alpha}=\iint v_{\eta} \delta\left(\mathbf{\Omega}-\mathbf{\Omega}^{\prime}\right) \delta\left(E-E^{\prime}\right) \Sigma_{\alpha, \eta} \varphi_{\alpha}\left(\mathbf{r}, \mathbf{\Omega}^{\prime}, E^{\prime}\right) d \mathbf{\Omega}^{\prime} d E^{\prime}
$$

The term

$$
v_{\eta}=\frac{\eta+1}{\eta}>0
$$

can be interpreted as the average number of (copy) neutrons produced by the $\alpha$-production operator having a delta spectrum (Zoia et al., 2015). Finally, the term $\lambda_{j} /\left(\lambda_{j}+\alpha\right)$ acts as a positive weight multiplier for the delayed neutrons (for physical reasons, the dominant eigenvalue satisfies $\alpha>-\min _{j}\left[\lambda_{j}\right]$ ). Several other techniques exist for $\alpha$ eigenvalue problems including neutrons and precursors, such as those suggested in (Hoogenboom, 2002; Nauchi, 2013; Josey, 2018): in this work, we will focus on the $\alpha$ - $k$ power iteration; method-to-method comparisons are left for future work.

\subsection{Generalized Iterated Fission Probability}

The equation adjoint to Eq. (5) reads (Bell and Glasstone, 1970)

$$
\frac{\alpha}{v} \varphi_{\alpha}^{\dagger}(\mathbf{r}, \mathbf{\Omega}, E)+\mathcal{L}^{\dagger} \varphi_{\alpha}^{\dagger}(\mathbf{r}, \mathbf{\Omega}, E)=\mathcal{F}_{p}^{\dagger} \varphi_{\alpha}^{\dagger}(\mathbf{r}, \boldsymbol{\Omega}, E)+\sum_{j} \frac{\lambda_{j}}{\lambda_{j}+\alpha} \mathcal{F}_{d, j}^{\dagger} \varphi_{\alpha}^{\dagger}(\mathbf{r}, \mathbf{\Omega}, E)
$$


where $\varphi_{\alpha}^{\dagger}$ is formally the eigenfunction adjoint to $\varphi_{\alpha}$ and physically represents the asymptotic importance ${ }^{1}$ of a neutron introduced into the system with coordinates $\mathbf{r}, \boldsymbol{\Omega}, E$. The adjoint operators appearing in Eq. (10) are defined as

$$
\begin{aligned}
& \mathcal{L}^{\dagger} \varphi_{\alpha}^{\dagger}(\mathbf{r}, \boldsymbol{\Omega}, E)=-\mathbf{\Omega} \cdot \nabla \varphi_{\alpha}^{\dagger}+\Sigma_{t} \varphi_{\alpha}^{\dagger}-\iint \Sigma_{s}\left(\mathbf{r}, \mathbf{\Omega}, E \rightarrow \mathbf{\Omega}^{\prime}, E^{\prime}\right) \varphi_{\alpha}^{\dagger}\left(\mathbf{r}, \boldsymbol{\Omega}^{\prime}, E^{\prime}\right) d \mathbf{\Omega}^{\prime} d E^{\prime} \\
& \mathcal{F}_{p}^{\dagger} \varphi_{\alpha}^{\dagger}(\mathbf{r}, \boldsymbol{\Omega}, E)=v_{p}(E) \Sigma_{f}(\mathbf{r}, E) \iint \frac{\chi_{p}\left(\mathbf{r}, E^{\prime}\right)}{4 \pi} \varphi_{\alpha}^{\dagger}\left(\mathbf{r}, \mathbf{\Omega}^{\prime}, E^{\prime}\right) d \mathbf{\Omega}^{\prime} d E^{\prime} \\
& \mathcal{F}_{d, j}^{\dagger} \varphi_{\alpha}^{\dagger}(\mathbf{r}, \boldsymbol{\Omega}, E)=v_{d, j}(E) \Sigma_{f}(\mathbf{r}, E) \iint \frac{\chi_{d, j}\left(\mathbf{r}, E^{\prime}\right)}{4 \pi} \varphi_{\alpha}^{\dagger}\left(\mathbf{r}, \mathbf{\Omega}^{\prime}, E^{\prime}\right) d \mathbf{\Omega}^{\prime} d E^{\prime}
\end{aligned}
$$

In a recent work, a Generalized IFP method has been proposed in order to compute $\varphi_{\alpha}^{\dagger}$ by Monte Carlo methods (Terranova et al., 2017). In the following we will briefly recall the Generalized IFP algorithm, whose basic idea is to relate the fundamental adjoint eigenfunction to the neutron importance, similarly to what is done for the regular $k$-eigenvalue IFP formulation (for a comprehensive description, see (Terranova et al., 2017)). The neutron importance $I_{\alpha}$ in the $\alpha$ eigenvalue problems can be estimated by recording the descendants after $M$ latent generations corresponding to an ancestor injected into the system at coordinates $\mathbf{r}, \boldsymbol{\Omega}, E$. For positive $\alpha$, the additional term $\Sigma_{\alpha}$ acts as a sterile capture: neutrons will not further contribute to the importance. Neutrons can contribute to the importance only by being promoted to the next generation by prompt and delayed fission ${ }^{2}$. For negative $\alpha$, neutrons can be also promoted to the next generation (and thus contribute to the importance $I_{\alpha}$ ) via the $\alpha$-production term, associated to the copy operator with cross section $\Sigma_{\alpha, \eta}$. Extensive comparisons between $\varphi_{\alpha}^{\dagger}$ as computed by estimating $I_{\alpha}$ via the Generalized IFP methods and by estimating the

\footnotetext{
${ }^{1}$ For very long times.

${ }^{2}$ In this latter case, their weight is assigned a correction factor $\lambda_{j} /\left(\lambda_{j}+\alpha\right)$, as discussed above.
} 
asymptotic importance in time-dependent simulations have been recently carried out and show that the two quantities are in very good agreement (Nauchi et al., 2019).

In order to estimate more generally bi-linear forms requiring both $\varphi_{\alpha}$ and $\varphi_{\alpha}^{\dagger}$, the $\alpha$ $k$ power iteration is first run until convergence is attained: after $D$ inactive cycles, neutrons will obey the fundamental eigenmode $\varphi_{\alpha}$. Then, at each successive generation $g$ a neutron is tagged as an ancestor, and its future contributions to the importance $I_{\alpha}$ are monitored. The corresponding importance $I_{\alpha} \propto \varphi_{\alpha}^{\dagger}$ of this ancestor is estimated at a later generation $g+M+1$ via the Generalized IFP method. Finally, adjoint-weighted tallies can be constructed for a generic operator $\mathcal{A}$ as

$$
\left\langle\varphi_{\alpha}^{\dagger} \mathcal{A} \varphi_{\alpha}\right\rangle \propto \sum_{i} \pi_{i} T_{i}
$$

Here $T_{i}$ is the tally associated to $\mathcal{A} \varphi_{\alpha}$ in the Monte Carlo simulation, evaluated during the first generation of the Generalized IFP cycle via the contribution of the ancestor; $\pi_{i}$ is the corresponding importance collected at the end of the Generalized IFP cycle, i.e., the number of descendant neutrons corresponding to the ancestor, after $M$ latent generations (Terranova et al., 2017); and the sum is extended over the ancestors, which bears a strong similarity to the procedure for $k$ eigenvalues (Kiedrowski et al., 2011). Contrary to the standard IFP method, where descendant neutrons can only originate from fission events, in the Generalized IFP method the $\alpha$-production can also promote particles to the next generation (for negative $\alpha$ ), and the contribution of the delayed fission neutrons to the next generation must be rescaled by a factor $\lambda_{j} /\left(\lambda_{j}+\alpha\right)$. The scheme of the Generalized IFP for bi-linear forms is displayed in Fig. 1. The error in the Generalized IFP calculations is estimated similarly to regular IFP, by collecting adjoint-weighted scores at each cycle after convergence (Terranova et al., 2017). In addition to the 
random nature of the simulated histories and the associated tallies $T_{i}$, fluctuations on the obtained averages stem from the variability of the neutron importances $\pi_{i}$ and of the $\alpha$ value estimated at each cycle.

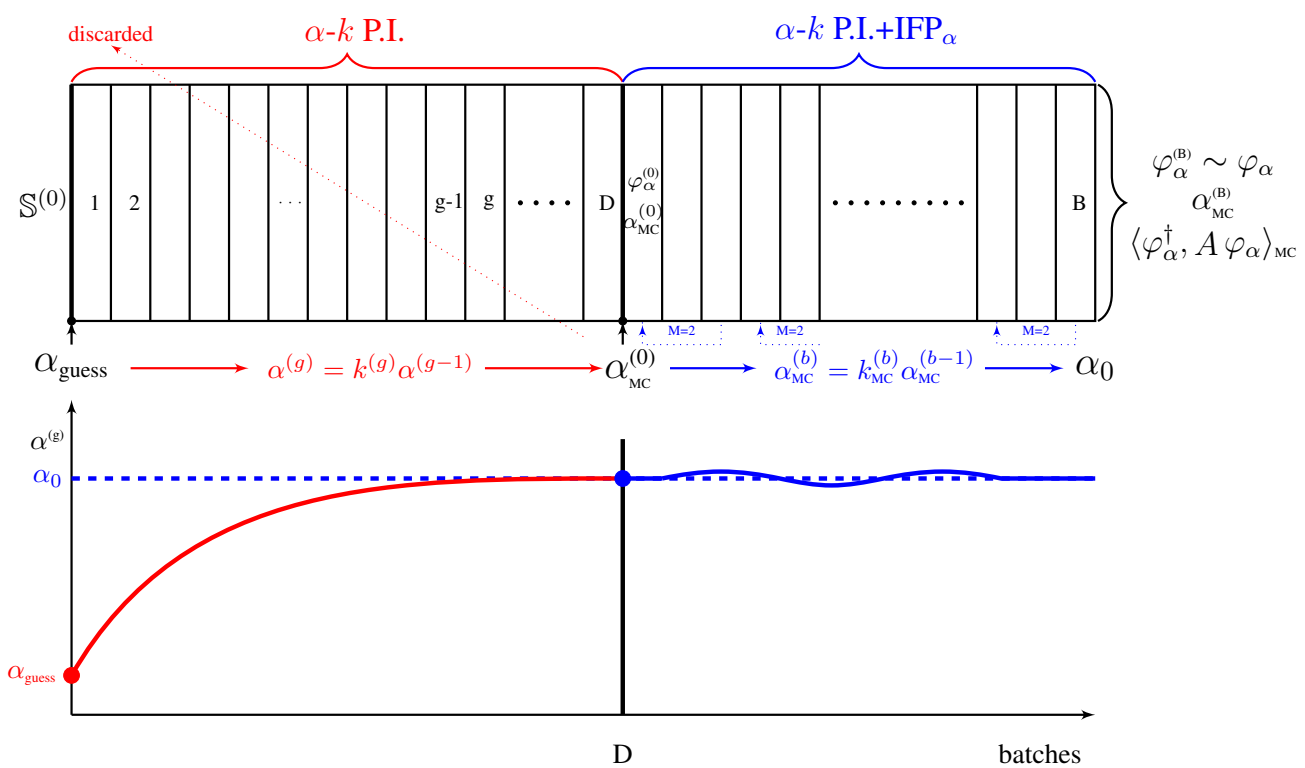

Figure 1: A schematic representation of the Generalized IFP algorithm for bi-linear forms $\left\langle\varphi_{\alpha}^{\dagger}, \mathcal{A} \varphi_{\alpha}\right\rangle$ within power iteration (P.I.). Courtesy of N. Terranova and A. Zoia, from Ref. (Terranova et al., 2017).

\section{First-order perturbation theory for time-eigenvalues}

In close analogy with the standard perturbation theory for $k$-eigenvalues, the firstorder perturbation theory for time-eigenvalues can be formulated as follows (Bell and Glasstone, 1970; Endo and Yamamoto, 2018). Consider Eqs. (5) and (10) for the direct and adjoint neutron flux, respectively, with leakage boundary conditions, and assume that a perturbation is introduced on the system parameters (cross sections, neutron multiplicities, decay constants, concentrations and/or densities). 
Let us denote by $\mathcal{A}^{\prime}=\mathcal{A}+\Delta \mathcal{A}$ the perturbed operators and by $\varphi_{\alpha}^{\prime}=\varphi_{\alpha}+\Delta \varphi_{\alpha}$ the corresponding perturbed neutron flux, which satisfies

$$
\frac{\alpha^{\prime}}{v} \varphi_{\alpha}^{\prime}(\mathbf{r}, \boldsymbol{\Omega}, E)+\mathcal{L}^{\prime} \varphi_{\alpha}^{\prime}(\mathbf{r}, \boldsymbol{\Omega}, E)=\mathcal{F}_{p}^{\prime} \varphi_{\alpha}^{\prime}(\mathbf{r}, \boldsymbol{\Omega}, E)+\sum_{j} \frac{\lambda_{j}^{\prime}}{\lambda_{j}^{\prime}+\alpha^{\prime}} \mathcal{F}_{d, j}^{\prime} \varphi_{\alpha}^{\prime}(\mathbf{r}, \boldsymbol{\Omega}, E),
$$

$\alpha^{\prime}=\alpha+\Delta \alpha$ being the perturbed eigenvalues. The key goal of perturbation theory is to express the variation $\Delta \alpha$ of the eigenvalue as a function of the variations introduced in the nuclear data. Multiply now Eq. (15) by the unperturbed adjoint flux $\varphi_{\alpha}^{\dagger}$ and Eq. (10) by the perturbed direct flux $\varphi_{\alpha}^{\prime}$, and integrate over the phase space (such integration will be denoted by brackets, namely, $\langle\cdots\rangle$ ). Subtracting the resulting equations yields

$$
\left(\alpha^{\prime}-\alpha\right)\left\langle\varphi_{\alpha}^{\dagger} \frac{1}{v} \varphi_{\alpha}^{\prime}\right\rangle=\left\langle\varphi_{\alpha}^{\dagger}\left(\Delta \mathcal{F}_{p}+\Delta \mathcal{S}-\Delta \Sigma_{t}\right) \varphi_{\alpha}^{\prime}\right\rangle+\left\langle\varphi_{\alpha}^{\dagger} \sum_{j} \Delta\left(\frac{\lambda_{j}}{\lambda_{j}+\alpha} \mathcal{F}_{d, j}\right) \varphi_{\alpha}^{\prime}\right\rangle,
$$

where we denote by $\mathcal{S}$ the scattering operator and we define the differences of operators

$$
\begin{aligned}
\Delta \mathcal{S} & =\mathcal{S}^{\prime}-\mathcal{S} \\
\Delta \Sigma_{t} & =\Sigma_{t}^{\prime}-\Sigma_{t} \\
\Delta \mathcal{F}_{p} & =\mathcal{F}_{p}^{\prime}-\mathcal{F}_{p} \\
\Delta\left(\frac{\lambda_{j}}{\lambda_{j}+\alpha} \mathcal{F}_{d, j}\right) & =\frac{\lambda_{j}^{\prime}}{\lambda_{j}^{\prime}+\alpha^{\prime}} \mathcal{F}_{d, j}^{\prime}-\frac{\lambda_{j}}{\lambda_{j}+\alpha} \mathcal{F}_{d, j} .
\end{aligned}
$$

Assuming then small perturbations and retaining only the first-order terms (products of perturbed quantities can be safely neglected ${ }^{3}$ ), we obtain the expression

\footnotetext{
${ }^{3}$ The first-order expansion of the scattering, collision and prompt fission operators works similarly as in the $k$-eigenvalue perturbation formulation. The expansion of the delayed fission operator on the other hand is unusual: for the sake of completeness, we provide its derivation.
} 
for $\Delta \alpha$, namely,

$$
\Delta \alpha=\frac{\left\langle\varphi_{\alpha}^{\dagger}\left(\Delta \mathcal{F}_{p}+\Delta \mathcal{S}-\Delta \Sigma_{t}\right) \varphi_{\alpha}\right\rangle+\sum_{j}\left(\frac{\lambda_{j}}{\lambda_{j}+\alpha}\left\langle\varphi_{\alpha}^{\dagger} \Delta \mathcal{F}_{d, j} \varphi_{\alpha}\right\rangle+\frac{\alpha \Delta \lambda_{j}}{\left(\lambda_{j}+\alpha\right)^{2}}\left\langle\varphi_{\alpha}^{\dagger} \mathcal{F}_{d, j} \varphi_{\alpha}\right\rangle\right)}{\left\langle\varphi_{\alpha}^{\dagger} \frac{1}{v} \varphi_{\alpha}\right\rangle+\sum_{j} \frac{\lambda_{j}}{\left(\lambda_{j}+\alpha\right)^{2}}\left\langle\varphi_{\alpha}^{\dagger} \mathcal{F}_{d, j} \varphi_{\alpha}\right\rangle} .
$$

An equivalent formulation ${ }^{4}$ was recently proposed in (Endo and Yamamoto, 2018; Yamamoto and Sakamoto, 2019).

The numerator of Eq. (22) contains two terms: the former expresses the variations in the prompt part of the Boltzmann operator $\Delta \mathcal{B}_{p}=\Delta \mathcal{F}_{p}+\Delta \mathcal{S}-\Delta \Sigma_{t}$, and is similar to the analogous expression appearing in the $k$-eigenvalue perturbation formula, whereas the latter expresses the variations in the delayed neutron contributions, either via the delayed fission operator itself, or via the precursor decay constants. The effect on the delayed fission operator is similar to that of the $k$-eigenvalue formulation, with the addition of a weight correction factor that is specific to the $\alpha$-eigenvalue formulation. The effect on the precursor decay constants, on the contrary, is a distinct feature of the $\alpha$-eigenvalue formulation: the $k$-eigenvalue is insensitive to variations in the $\lambda_{j}$. The denominator of Eq. (22) can be also given a suggestive interpretation: the former term clearly represents the adjoint-weighted time spent by the neutrons in the system (Bell and Glasstone, 1970). By using the ${\overline{\text { Inserting }} \alpha^{\prime}=\alpha+\Delta \alpha \text { and } \lambda_{j}^{\prime}=\lambda_{j}+\Delta \lambda_{j}}_{j}$ into Eq. (20), using the expansion $1 /(1+x)=1-x+\cdots$ and keeping only the first-order terms yields

$$
\begin{aligned}
\Delta\left(\frac{\lambda_{j}}{\lambda_{j}+\alpha} \mathcal{F}_{d, j}\right) & =\frac{\lambda_{j}+\Delta \lambda_{j}}{\lambda_{j}+\alpha+\Delta \lambda_{j}+\Delta \alpha}\left(\mathcal{F}_{d, j}+\Delta \mathcal{F}_{d, j}\right)-\frac{\lambda_{j}}{\lambda_{j}+\alpha}\left(\mathcal{F}_{d, j}\right) \\
& =\frac{\lambda_{j}}{\lambda_{j}+\alpha}\left(1+\frac{\Delta \lambda_{j}}{\lambda_{j}}\right)\left(1-\frac{\Delta \lambda_{j}+\Delta \alpha}{\lambda_{j}+\alpha}\right)\left(\mathcal{F}_{d, j}+\Delta \mathcal{F}_{d, j}\right)-\frac{\lambda_{j}}{\lambda_{j}+\alpha} \mathcal{F}_{d, j} \\
& =\frac{\lambda_{j}}{\lambda_{j}+\alpha} \Delta \mathcal{F}_{d, j}+\frac{\alpha \Delta \lambda_{j}}{\left(\lambda_{j}+\alpha\right)^{2}} \mathcal{F}_{d, j}-\frac{\lambda_{j} \Delta \alpha}{\left(\lambda_{j}+\alpha\right)^{2}} \mathcal{F}_{d, j} .
\end{aligned}
$$

${ }^{4}$ Contrary to (Endo and Yamamoto, 2018), we neglect the perturbation on the neutron speed. 
definitions

$$
c_{\alpha, j}(\mathbf{r})=\frac{1}{\lambda_{j}+\alpha} \iint v_{d, j}\left(E^{\prime}\right) \Sigma_{f}\left(\mathbf{r}, E^{\prime}\right) \varphi_{\alpha}\left(\mathbf{r}, \mathbf{\Omega}^{\prime}, E^{\prime}\right) d \mathbf{\Omega}^{\prime} d E^{\prime}
$$

and

$$
c_{\alpha, j}^{\dagger}(\mathbf{r})=\frac{\lambda_{j}}{\lambda_{j}+\alpha} \iint \frac{\chi_{d, j}\left(\mathbf{r}, E^{\prime}\right)}{4 \pi} \varphi_{\alpha}^{\dagger}\left(\mathbf{r}, \mathbf{\Omega}^{\prime}, E^{\prime}\right) d \mathbf{\Omega}^{\prime} d E^{\prime}
$$

for the direct and adjoint precursor eigenmodes, respectively, we can rewrite

$$
\frac{\lambda_{j}}{\left(\lambda_{j}+\alpha\right)^{2}}\left\langle\varphi_{\alpha}^{\dagger} \mathcal{F}_{d, j} \varphi_{\alpha}\right\rangle=\left\langle c_{\alpha, j}^{\dagger}(\mathbf{r}) c_{\alpha, j}(\mathbf{r})\right\rangle,
$$

carrying the units of a time. The denominator of Eq. (22) might be thus thought of as an adjoint-weighted average life-time accounting for both neutron and precursors within the system.

The fundamental $\alpha$ eigenvalue represents the inverse of the asymptotic reactor period $T$, including the precursor contributions. It is then interesting to express the first-order perturbation $\Delta T$ of the reactor period in terms of the perturbation $\Delta \alpha$ of the eigenvalue: by using $T=1 / \alpha$, we find

$$
\Delta T=-\frac{\Delta \alpha}{\alpha^{2}} .
$$

The effects of a perturbation of nuclear data on the prompt time eigenvalue $\alpha_{p}$, which results from Eqs. (5) and (10) when the delayed contributions are neglected, can be estimated by using the same argument as above. The resulting expression for $\Delta \alpha_{p}$ is simpler (Bell and Glasstone, 1970), and can be written as

$$
\Delta \alpha_{p}=\frac{\left\langle\varphi_{\alpha}^{\dagger}\left(\Delta \mathcal{F}_{p}+\Delta \mathcal{S}-\Delta \Sigma_{t}\right) \varphi_{\alpha}\right\rangle}{\left\langle\varphi_{\alpha}^{\dagger} \frac{1}{v} \varphi_{\alpha}\right\rangle}=\frac{\left\langle\varphi_{\alpha}^{\dagger} \Delta \mathcal{B}_{p} \varphi_{\alpha}\right\rangle}{\left\langle\varphi_{\alpha}^{\dagger} \frac{1}{v} \varphi_{\alpha}\right\rangle}
$$

which physically represents the (adjoint-weighted) total variation of the events occurring during the (prompt) neutron histories over the (adjoint-weighted) neutron life-time (Endo and Yamamoto, 2018). 


\subsection{Estimating the perturbations by Monte Carlo methods}

The operators $\Delta S, \Delta \mathcal{F}_{p}, \Delta \Sigma_{t}, \Delta \mathcal{F}_{d, j}$ and $\Delta \lambda_{j}$ are independent of $\alpha$ and can be analytically expressed in terms of the variations of nuclear data, concentrations and densities. As discussed above, the Generalized IFP method can be indifferently applied to sub- and super-critical systems (corresponding to negative and positive dominant time eigenvalue, respectively). With the exception of $\Delta \lambda_{j}$, these operators are the same as those occurring in the SPT formulation for $k$-eigenvalue problems. As such, the bi-linear forms appearing in Eq. (22) can be computed based on the tallies proposed in (Kiedrowski et al., 2011) for standard IFP-based reactivity perturbations, the only difference being that the particle trajectories $\tau$ must be simulated within the Generalized IFP scheme described above. The explicit expressions for theses estimators are recalled in Tab. 1.

\begin{tabular}{cc}
\hline Scalar product & Estimators $T_{i}$ \\
\hline$\left\langle\varphi_{\alpha}^{\dagger} \Delta \Sigma_{t} \varphi_{\alpha}\right\rangle$ & $\sum_{\tau \in i} \Delta \Sigma_{t} \ell_{\tau}$ \\
$\left\langle\varphi_{\alpha}^{\dagger} \Delta \mathcal{S} \varphi_{\alpha}\right\rangle$ & $\sum_{\tau \in i} \frac{\Delta \Sigma_{s}}{\Sigma_{s}}$ \\
$\left\langle\varphi_{\alpha}^{\dagger} \Delta \mathcal{F}_{p} \varphi_{\alpha}\right\rangle$ & $\frac{\Delta\left(v_{p} \Sigma_{f}\right)}{v_{p} \Sigma_{f}}$ \\
$\frac{\lambda_{j}}{\lambda_{j}+\alpha}\left\langle\varphi_{\alpha}^{\dagger} \Delta \mathcal{F}_{d, j} \varphi_{\alpha}\right\rangle$ & $\frac{\Delta\left(v_{d, j} \Sigma_{f}\right)}{v_{d, j} \Sigma_{f}}$ \\
$\left\langle\varphi_{\alpha}^{\dagger} \frac{1}{v} \varphi_{\alpha}\right\rangle$ & $\sum_{\tau \in i} \frac{1}{v} \ell_{\tau}$ \\
\hline
\end{tabular}

Table 1: Monte Carlo estimators for perturbed operators. Notation is as in (Terranova et al., 2018) and $\ell_{\tau}$ is the length travelled by the neutron during history $\tau$.

The operator $\Delta \lambda_{j}$ is easily expressed as the difference between the perturbed and unperturbed decay constants. Care must be taken in evaluating the delayed neutron contributions $\pi_{i}^{d, j}$ to the neutron importance of family $j$ : since each delayed neutron promoted to the following generation is attributed a weight 
correction factor $\lambda_{j} /\left(\lambda_{j}+\alpha\right)$, we have

$$
\frac{\lambda_{j}}{\lambda_{j}+\alpha}\left\langle\varphi_{\alpha}^{\dagger} \mathcal{F}_{d, j} \varphi_{\alpha}\right\rangle \propto \sum_{i} \pi_{i}^{d, j}
$$

contrary to the case of $k$-eigenvalue IFP calculations, where the estimator associated to the delayed fission operator is simply $\pi_{i}^{d, j}$.

\section{Sensitivity analysis for time eigenvalues}

In uncertainty propagation and bias projection, it may be easier to work with sensitivity coefficients rather than perturbations. By using the same arguments as in (Endo and Yamamoto, 2018), first-order sensitivity coefficients of the $\alpha$ eigenvalue to a generic nuclear data parameter $x_{n}$ can be derived based on a slight modification of Eq. (22), which yields

$$
S_{\alpha, x_{n}}=\frac{x_{n}}{\alpha} \frac{\partial \alpha}{\partial x_{n}}=\frac{1}{\alpha} \frac{\left\langle\varphi_{\alpha}^{\dagger} \partial_{x_{n}} \mathcal{B}_{p} \varphi_{\alpha}\right\rangle+\sum_{j}\left(\frac{\lambda_{j}}{\lambda_{j}+\alpha}\left\langle\varphi_{\alpha}^{\dagger} \partial_{x_{n}} \mathcal{F}_{d, j} \varphi_{\alpha}\right\rangle+\frac{\alpha \partial_{x_{n}} \lambda_{j}}{\left(\lambda_{j}+\alpha\right)^{2}}\left\langle\varphi_{\alpha}^{\dagger} \mathcal{F}_{d, j} \varphi_{\alpha}\right\rangle\right)}{\left\langle\varphi_{\alpha}^{\dagger} \frac{1}{v} \varphi_{\alpha}\right\rangle+\sum_{j} \frac{\lambda_{j}}{\left(\lambda_{j}+\alpha\right)^{2}}\left\langle\varphi_{\alpha}^{\dagger} \mathcal{F}_{d, j} \varphi_{\alpha}\right\rangle}
$$

where $\partial_{x_{n}}$ applied to a generic operator $\mathcal{A}$ denotes the scaled partial derivative

$$
\partial_{x_{n}} \mathcal{A}=x_{n} \frac{\partial \mathcal{A}}{\partial x_{n}} .
$$

\subsection{Estimating the sensitivity coefficients by Monte Carlo methods}

In the following we will discuss the Monte Carlo estimators associated to the terms appearing in Eq. (29). Once the random contributions $T_{i}$ for the derivative of each operator with respect to the parameter $x_{n}$ have been collected, the corresponding adjoint-weighted quantities can be obtained as in Sec. 2.1 by multiplying $T_{i}$ by the associated importance $\pi_{i}$ at then end of each Generalized IFP cycle. Again, the Generalized IFP method can be indifferently applied to sub- and super-critical systems. 
The denominator appearing in Eq. (29) is exactly the same as that occurring for perturbations, and can be thus estimated as detailed above. The estimators needed for the terms appearing at the numerator in the expression of the $\alpha$-eigenvalue sensitivities can by estimated by adapting the IFP-based tallies presented in (Kiedrowski and Brown, 2013) for $k$-eigenvalue sensitivities. Table 2 displays the random contributions $T_{i}$ for sensitivity coefficients.

\begin{tabular}{rc}
\hline Scalar product & Estimators $T_{i}$ \\
\hline$\left\langle\varphi_{\alpha}^{\dagger} \partial_{x_{n}} \Sigma_{t} \varphi_{\alpha}\right\rangle$ & $\sum_{\tau \in i} \delta_{x_{n}, \Sigma_{t}} \delta_{E \in g} \gamma_{n} x_{n} \ell_{\tau}$ \\
$\left\langle\varphi_{\alpha}^{\dagger} \partial_{x_{n}} \mathcal{S} \varphi_{\alpha}\right\rangle$ & $\sum_{\tau \in i} \delta_{x_{n}, \sigma_{s}} \delta_{n, j} \delta_{E \in g}$ \\
$\left\langle\varphi_{\alpha}^{\dagger} \partial_{x_{n}} \mathcal{F}_{p} \varphi_{\alpha}\right\rangle$ & $\sum_{\tau \in i} \delta_{x_{n}, v_{p} \sigma_{f}} \delta_{n, j} \delta_{E \in g}$ \\
$\frac{\lambda_{j}}{\lambda_{j}+\alpha}\left\langle\varphi_{\alpha}^{\dagger} \partial_{x_{n}} \mathcal{F}_{d, j} \varphi_{\alpha}\right\rangle$ & $\sum_{\tau \in i} \delta_{x_{n}, v_{d, j}} \sigma_{f} \delta_{n, j} \delta_{E \in g}$ \\
$\frac{\alpha}{\left(\lambda_{j}+\alpha\right)^{2}} \partial_{x_{n}} \lambda_{j}\left\langle\varphi_{\alpha}^{\dagger} \mathcal{F}_{d, j} \varphi_{\alpha}\right\rangle$ & $\sum_{\tau \in i} \delta_{x_{n}, \lambda j} \delta_{n, j} \frac{\alpha}{\lambda_{j}+\alpha}$ \\
\hline
\end{tabular}

Table 2: Monte Carlo estimators for sensitivity coefficients. Notation is as in (Terranova et al., 2018), $\ell_{\tau}$ is the length travelled by the neutron during history $\tau$ and $\gamma_{n}$ is the concentration of the isotope related to $x_{n}$.

Similarly to perturbations, the variations in the prompt part of the Boltzmann operator is similar to the analogous expression appearing in the $k$-eigenvalue sensitivity coefficients. For the delayed fission operator contribution to the sensitivity for the precursor family $j, \delta_{x_{n}, v_{d, j} \sigma_{f}}$ is equal to one if the parameter $x_{n}$ is equal to a delayed fission for family $j$, cross section or average number of delayed neutrons $v_{d, j}$, and $\delta_{n, j}$ is equal to one when the sampled nucleus and fission reaction match the considered nucleus and reaction $n$. The random contribution is set to zero otherwise. The factor $\lambda_{j} /\left(\lambda_{j}+\alpha\right)$ is actually implicitly taken into account, as mentioned above for the perturbation tallies.

Considering the decay constant contributions to the sensitivity corresponding to 15 
the precursor family $j, \delta_{x_{n}, \lambda^{j}}$ is equal to one if the parameter $x_{n}$ is equal to the decay constant $\lambda_{j}$ of the precursor family $j$ and $\delta_{n, j}$ is equal to one when the sampled nucleus and fission reaction match the considered nucleus and reaction $n$. The random contribution is set to zero otherwise. Again, a factor $\lambda_{j} /\left(\lambda_{j}+\alpha\right)$ is implicitly estimated by the considered tally.

Finally, constrained and unconstrained sensitivities to fission spectrum and scattering laws can be computed similarly to $k$-eigenvalue sensitivities (the $\lambda_{j} /\left(\lambda_{j}+\alpha\right)$ factor will be implicitly taken into account for delayed fission contributions to the fission spectrum).

\subsection{Normalization of the sensitivity coefficients}

The sensitivity coefficients given in Eq. (29) are relative, as customary for $k$ eigenvalue sensitivity analysis. This implies that the amplitude of $S_{\alpha, x_{n}}$ depends on the (absolute) value of $\alpha$, whose estimates may in turn be substantially affected by the choice of nuclear data libraries (and phase space discretization in the case of deterministic solvers). These considerations are of utmost importance when performing code-to-code comparisons for the sensitivity coefficients. For instance, a difference of about $150 \mathrm{pcm}$ on the estimated reactivity between a Monte Carlo and a deterministic code might correspond to a discrepancy of about $25 \%$ in the prompt neutron decay constant value $\alpha_{p}$, as illustrated in Sec. 5.3. In such cases, it is reasonable to rescale the computed sensitivity coefficients by the ratio of $\alpha$ values estimated by the different codes. In addition to these considerations, the definition in Eq. (29) may lead to a singularity when the system is exactly critical $(\alpha=0)$. These two issues may be overcome by considering non-normalized coefficients

$$
\tilde{S}_{\alpha, x_{n}}=x_{n} \frac{\partial \alpha}{\partial x_{n}}
$$


The Monte Carlo estimators would not be affected by this definition, apart from an overall scaling factor $1 / \alpha$.

\section{Verification tests}

The algorithms described in Sec. 3 and 4 have been implemented in the development version of TRIPOLI- $4{ }^{\circledR}$. For the verification of the proposed methods for first-order perturbations and sensitivities of $\alpha$-eigenvalues, we have selected three significant benchmark configurations. The first is a continuous-energy neutron thermalization problem in an infinite medium, for which reference analytic solutions can be obtained. The second is a two energy groups and two precursor families transport problem in an infinite medium, which can be solved analytically for the $k$ - and $\alpha$-eigenvalue formulations (Terranova et al., 2017; Kiedrowski, 2010). Finally, we have numerically verified our implementation by considering the HEU-SOL-THERM-012 benchmark, for which sensitivity profiles have been recently computed in (Endo and Yamamoto, 2018) by using a deterministic solver. All results provided in the following tables are presented with a one- $\sigma$ Monte Carlo relative uncertainty in \%. For energy-resolved sensitivity profiles, the uncertainty displayed in the figures corresponds to one absolute standard deviation.

\subsection{A neutron thermalization model}

We have considered a simplified continuous-energy neutron thermalization model, describing an infinite medium composed of an ideal gas of non-fissile nuclei, which lends itself to exact solutions for the $\alpha$-eigenvalue equation (Duderstadt and Martin, 1979). Scattering is assumed to be at the thermal equilibrium, with a so-called amnesia kernel that is capable of thermalizing the neutrons in a single collision, with a Maxwellian spectrum $M(E) \propto \sqrt{E} \exp (-E / \theta), \theta$ being the 
average post-collision energy. In addition to scattering, we take into account capture, which leads to the eigenvalue problem

$$
\frac{\alpha}{v(E)} \varphi_{\alpha}(E)+\Sigma_{t}(E) \varphi_{\alpha}=M(E) \int_{0}^{\infty} \Sigma_{s}\left(E^{\prime}\right) \varphi_{\alpha}\left(E^{\prime}\right) d E^{\prime},
$$

where $v=\sqrt{E}$ by conveniently choosing the units of the speed ${ }^{5}$. The normalization of $\varphi_{\alpha}(E)$ is arbitrary, and it is convenient to set

$$
\int_{0}^{\infty} \Sigma_{s}\left(E^{\prime}\right) \varphi_{\alpha}\left(E^{\prime}\right) d E^{\prime}=1
$$

We set now the cross sections

$$
\begin{aligned}
& \Sigma_{s}(E)=\Sigma_{s}^{0} \\
& \Sigma_{c}(E)=\frac{\Sigma_{c}^{0}}{\sqrt{E}}
\end{aligned}
$$

and choose $\theta=1$. By using the same strategy as in (Duderstadt and Martin, 1979), it can be shown that the dominant $\alpha$ eigenvalue reads then $\alpha=-\Sigma_{c}^{0}$, with associated eigenfunction

$$
\varphi_{\alpha}=\frac{M(E)}{\Sigma_{s}^{0}}
$$

By considering the equation adjoint to Eq. (32) and using the normalization

$$
\int_{0}^{\infty} M\left(E^{\prime}\right) \varphi_{\alpha}^{\dagger}\left(E^{\prime}\right) d E^{\prime}=1
$$

the adjoint flux turns out to be simply $\varphi_{\alpha}^{\dagger}=1$ for this problem.

The sensitivity coefficients of the time eigenvalue with respect to the capture cross section can thus be computed exactly, based on Eq. (29): for an arbitrary energy

\footnotetext{
${ }^{5}$ The energy is equally expressed in arbitrary units, and the typical scale of energy is implicitly defined by setting the value of the average post-collision energy $\theta$.
} 
group between energies $E_{g}$ and $E_{g+1}$ we have

$$
S_{g}\left(\Sigma_{c}\right)=-\frac{1}{\alpha} \frac{\int_{E_{g}}^{E_{g+1}} \varphi_{\alpha}^{\dagger}\left(E^{\prime}\right) \Sigma_{c}\left(E^{\prime}\right) \varphi_{\alpha}\left(E^{\prime}\right) d E^{\prime}}{\int_{0}^{\infty} \varphi_{\alpha_{0}}^{\dagger}\left(E^{\prime}\right) \frac{1}{v^{\prime}} \varphi_{\alpha}\left(E^{\prime}\right) d E^{\prime}}=\exp \left(-E_{g}\right)-\exp \left(-E_{g+1}\right)
$$

The sensitivity coefficients computed by the Generalized IFP method described in Sec. 4 are compared to Eq. (36) in Fig. 2: a very good agreement is found. Higher fluctuations are found for $E \gg \theta$, due to poor statistics in the tails of the post-scattering distribution.

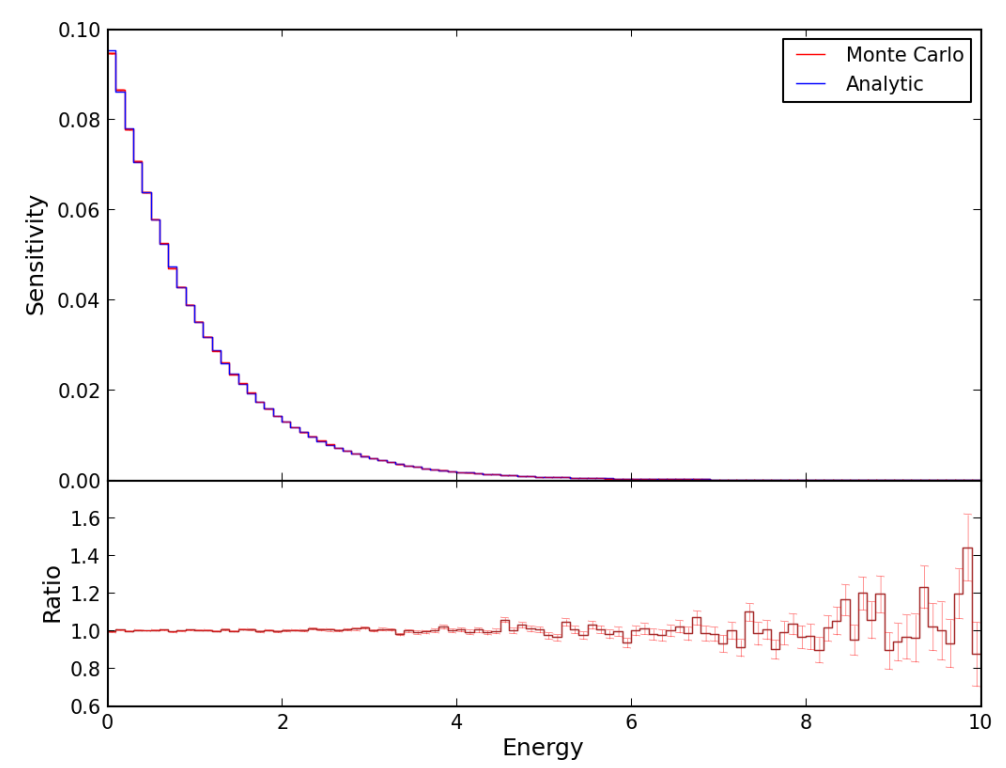

Figure 2: Sensitivity profiles to absorption cross section computed using Monte Carlo Generalized IFP (red) and analytic solutions (blue).

\subsection{Two groups infinite medium}

As a second verification test, we have considered a two-group infinite medium configuration, for which exact solutions can be computed for the direct and adjoint time eigenpairs (Terranova et al., 2017). The model assumes that there is no upscattering, neutrons can only induce fissions when colliding in the thermal group 
and that all prompt fission neutrons are emitted exclusively in $g=1$. Two delayed families $a$ and $b$ are taken into account. The resulting eigenmodes read

$$
\begin{aligned}
\frac{\varphi_{\alpha, 2}}{\varphi_{\alpha, 1}} & =\frac{\Sigma_{s, 12}}{\frac{\alpha}{v_{2}}+\Sigma_{r, 2}-\zeta_{2} v_{\mathrm{f}, 2} \Sigma_{\mathrm{f}, 2}} \\
\frac{\varphi_{\alpha, 1}^{\dagger}}{\varphi_{\alpha, 2}^{\dagger}} & =\frac{\Sigma_{s, 12}}{\frac{\alpha}{v_{1}}+\Sigma_{r, 1}} \\
\zeta_{i} & =\sum_{j} \frac{\lambda_{j}}{\lambda_{j}+\alpha} \chi_{d, j}\left(v_{i}\right) \beta_{j},
\end{aligned}
$$

where $\Sigma_{r}=\Sigma_{c}+\Sigma_{f}$ is the removal cross section and the $\alpha$ eigenvalues are the roots of a fourth-order polynomial (Terranova et al., 2017). The model parameters are provided in Tab. 3. In order to test our implementation for negative $\alpha$-eigenvalues, the thermal capture cross section was increased by $50 \%$ with respect to its reference value given in (Terranova et al., 2017). The fundamental eigenvalue corresponding to these parameters reads $\alpha_{0}=-0.03672$.

\begin{tabular}{ccccccccccccc}
\hline$g$ & $v$ & $\Sigma_{c, g}$ & $\Sigma_{\mathrm{f}, g}$ & $\Sigma_{\mathrm{s}, g \rightarrow 1}$ & $\Sigma_{\mathrm{s}, g \rightarrow 2}$ & $v_{t o t}$ & $\beta_{a}$ & $\beta_{b}$ & $\chi_{a \rightarrow g}$ & $\chi_{b \rightarrow g}$ & $\lambda_{a}$ & $\lambda_{b}$ \\
\hline 1 & 10 & 1 & 0 & $1 / 2$ & $1 / 2$ & 0 & 0 & 0 & $3 / 4$ & $1 / 2$ & & \\
2 & 5 & $3 / 2$ & 1 & 0 & 1 & $24 / 5$ & $1 / 4$ & $1 / 8$ & $1 / 4$ & $1 / 2$ & 0.5 & 0.07 \\
\hline
\end{tabular}

Table 3: Values for the physical parameters of the two-group infinite medium system

Reference values for the first-order perturbations and sensitivities can be then obtained from Eqs. (22) and (29) by using the expressions for the direct and adjoint fluxes given above. The perturbation contributions due to the collision term $\Delta \Sigma_{t}$, to scattering $\Delta \mathcal{S}$, to prompt fission $\Delta \mathcal{F}_{p}$, to delayed fission $\Delta \mathcal{F}_{d, j}$ and to the decay constants $\Delta \lambda_{j}$ can be separately estimated and contrasted to their analytical counterparts. For comparison, the exact perturbation $\Delta \alpha_{e x}$ is also obtained by analytically computing the $\alpha$ eigenvalue for the perturbed and 
reference configuration and taking the difference.

Table 4 shows the results for a $-10 \%$ perturbation of the fission cross section $\Sigma_{\mathrm{f}, 2}$ and a $+7 \%$ perturbation of the second family decay constant $\lambda_{b}$. Monte Carlo perturbation results obtained from the Generalized IFP method are in good agreement with analytical results, the discrepancy lying below $0.7 \%$ for all the contributions to the total perturbation. Although discrepancies lie slightly outside of the 3- $\sigma$ confidence interval, the standard deviation is known to be underestimated in the Monte Carlo $\alpha$ - $k$ power iteration due to cycle-to-cycle correlations (Zoia et al., 2014). For this configuration, the first-order perturbation is close to the exact perturbation, with a small discrepancy of $0.7 \%$.

\begin{tabular}{lccc}
\hline Perturbation term & Analytic & Monte Carlo $\pm \sigma(\%)$ & MC / Analytic \\
\hline Collision & -0.00370 & $-0.00372 \pm 0.13$ & 1.0053 \\
Prompt fission & -0.00371 & $-0.00374 \pm 0.17$ & 1.0066 \\
Delayed fission & -0.00552 & $-0.00554 \pm 0.09$ & 1.0040 \\
Decay constant & -0.00246 & $-0.00246 \pm 0.04$ & 0.9991 \\
Total first-order $\Delta \alpha$ & -0.00799 & $-0.00801 \pm 0.07$ & 1.0031 \\
Exact $\Delta \alpha$ & -0.00807 & & \\
\hline$\alpha$-eigenvalue & -0.03672 & $-0.03671 \pm 0.014$ & 0.9996 \\
\hline
\end{tabular}

Table 4: Results for the variation $\Delta \alpha$ in the time eigenvalue corresponding to a perturbation of both the fission cross section and $\lambda_{b}$ (the decay constant of the second precursor family).

The perturbation $\Delta \alpha$ corresponding to variations from $-10 \%$ to $+10 \%$ of the capture cross section in both energy groups is displayed in Fig. 3. A very good agreement is found between the reference solutions for the first-order perturbations and the Monte Carlo simulations. In the limit of small perturbations, the first-order 
$\Delta \alpha$ is close to the exact perturbation, as expected.

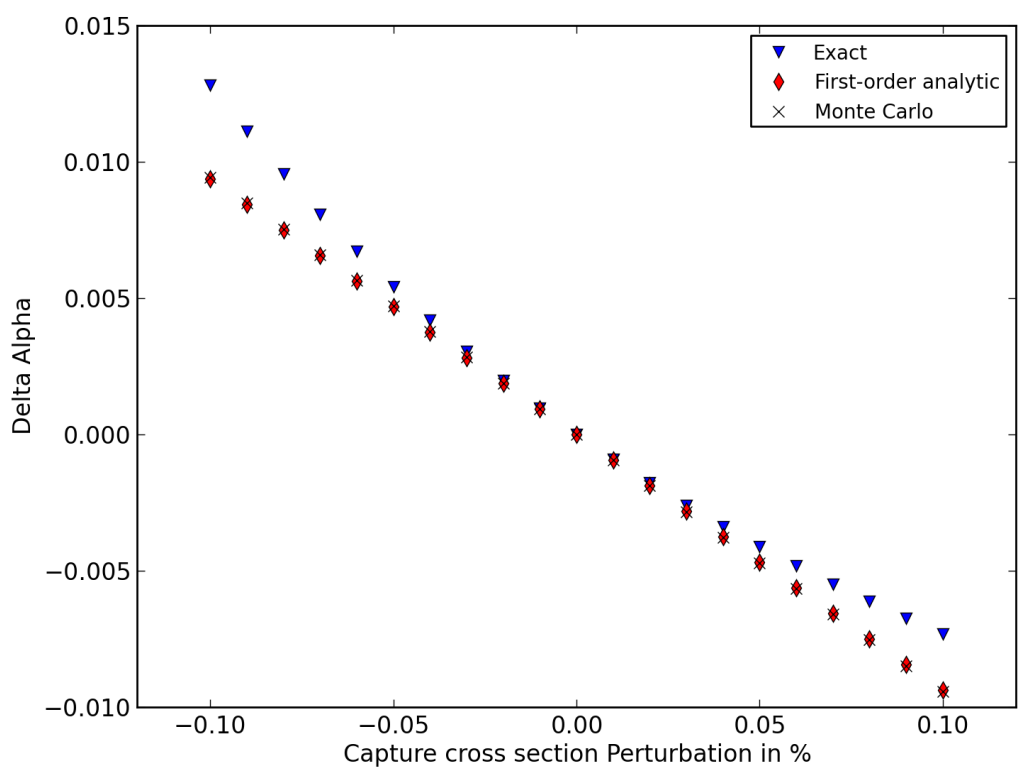

Figure 3: Change $\Delta \alpha$ of the time eigenvalue as a function of the capture cross section perturbation. We compare the first-order perturbations obtained by the Generalized IFP method (black crosses) to the first-order perturbation formula that is used as a reference (red diamonds). The results corresponding to the exact perturbation are also displayed (blue triangles), for comparison.

Table 5 displays the sensitivity coefficients of $\alpha$ with respect to various nuclear data, including cross sections and decay constants. A very good agreement is observed between the Monte Carlo and analytic calculations of sensitivity coefficients: discrepancies lie below $0.3 \%$ and analytic results lie within 3- $\sigma$ confidence intervals of the Monte Carlo results. 


\begin{tabular}{lccc}
\hline Cross section & Analytic & Monte Carlo $\pm \sigma(\%)$ & MC / Analytic \\
\hline$\Sigma_{c, 1}$ & 1.03673 & $1.03966 \pm 0.083$ & 1.00282 \\
$\Sigma_{c, 2}$ & 1.51308 & $1.51579 \pm 0.065$ & 1.00179 \\
$\Sigma_{f, 2}$ & -1.50568 & $-1.50864 \pm 0.061$ & 1.00197 \\
$\Sigma_{s, 1}$ & -1.03292 & $-1.03639 \pm 0.083$ & 1.00335 \\
$\lambda_{a}$ & 0.0518364 & $0.0519299 \pm 0.084$ & 1.00180 \\
$\lambda_{b}$ & 0.936949 & $0.936835 \pm 0.005$ & 0.99988 \\
\hline
\end{tabular}

Table 5: Sensitivity coefficients of the time eigenvalue $\alpha$ in the two-group infinite medium model.

\subsubsection{Prompt neutron decay constant}

We conclude the analysis of the two-group model by considering the prompt neutron decay constant, which results from turning off delayed neutrons $\left(\beta_{a}=\right.$ $\beta_{b}=0$ ) and setting the total $v_{t o t}$ to the prompt $v_{p}$. Keeping the other parameters of the model as in Tab. 3, the dominant time-eigenvalue reads $\alpha_{0}=-5$. Tables 6 and 7 display the sensitivity coefficients and perturbations, respectively, of the prompt neutron decay constant with respect to nuclear data.

\begin{tabular}{lccc}
\hline Cross section & Analytic & Monte Carlo $\pm \sigma(\%)$ & MC / Analytic \\
\hline$\Sigma_{c, 1}$ & 0.857143 & $0.857317 \pm 0.026$ & 1.00020 \\
$\Sigma_{c, 2}$ & 0.857143 & $0.856377 \pm 0.020$ & 0.99911 \\
$\Sigma_{f, 2}$ & -0.285714 & $-0.28616 \pm 0.115$ & 1.00156 \\
$\Sigma_{s, 1}$ & -0.428571 & $-0.427668 \pm 0.077$ & 0.99789 \\
\hline
\end{tabular}

Table 6: Sensitivity coefficients of the prompt decay constant $\alpha$ in the two-group infinite medium.

As above, results show a very good agreement between the Monte Carlo and analytic solutions. 


\begin{tabular}{lccc}
\hline Perturbation term & Analytic & Monte Carlo $\pm \sigma(\%)$ & $\mathrm{MC} /$ Analytic \\
\hline Collision & -0.285714 & $-0.28558 \pm 0.018$ & 0.99953 \\
Prompt fission & -0.428571 & $-0.428713 \pm 0.033$ & 1.00033 \\
Total first-order $\Delta \alpha$ & -0.142857 & $-0.143133 \pm 0.113$ & 1.00193 \\
Exact $\Delta \alpha$ & -0.14835 & & \\
\hline$\alpha$-eigenvalue & -5. & $-5.00226 \pm 0.0092$ & 1.00052 \\
\hline
\end{tabular}

Table 7: Results for the variation $\Delta \alpha$ of the prompt decay constant corresponding to a perturbation of $-10 \%$ of the fission cross section in the two-group infinite medium.

\subsection{HEU-SOL-THERM-012}

As a final verification case for continuous-energy transport, we have selected the HEU-SOL-THERM-012 benchmark, taken from the ICSBEP handbook (ICSBEP, 2018). The chosen configuration corresponds to the simplified version of this benchmark, with three concentric spheres containing respectively a $\mathrm{UO}_{2} \mathrm{~F}_{2}$ solution with highly enriched Uranium $(27.9244 \mathrm{~cm}$ radius), aluminium cladding (0.2 cm thickness) and water (15 cm thickness). The time-eigenvalue sensitivity analysis for the proposed model was carried out in (Endo and Yamamoto, 2018) by using a modified 1D deterministic solver of PARTISN (Alcouffe et al., 2018) with 252 energy groups (Rearden and Jessee, 2018) and the ENDF/B-VII.1 library (Chadwick et al., 2011). This offers the possibility of contrasting the results obtained by our continuous-energy implementation in TRIPOLI- $4{ }^{\circledR}$ to those of (Endo and Yamamoto, 2018). In (Endo and Yamamoto, 2018), the sensitivity analysis was performed on the prompt neutron decay constant $\alpha_{p}$. In Monte Carlo, this constant can be estimated by turning off the sampling of delayed neutrons. This is what is done in this configuration. Our calculations have been performed 
with 15000 neutrons per cycle, 1000 inactive cycles, 3000 active cycles and 10 latent generations for the Generalized IFP method. In view of performing consistent code-to-code comparisons, we have also selected the ENDF/B-VII.1 nuclear data library. Digitalized data of the deterministic calculations have been kindly provided by the authors of (Endo and Yamamoto, 2018).

A preliminary Monte Carlo run for time-eigenvalue calculations yields a prompt decay constant $\alpha_{p}=-76.77991 \mathrm{~s}^{-1} \pm 0.14102 \%$. This is consistent with the estimate $\tilde{\alpha}_{p} \simeq\left(\beta_{\text {eff }}-\rho\right) / \Lambda_{\text {eff }}$ that can be obtained from a $k$-eigenvalue calculation (Truchet et al., 2015), using the point-kinetics approximation with IFP-based values for kinetics parameters: the TRIPOLI- $4{ }^{\circledR}$ calculations yield $\beta_{\text {eff }}=714 \mathrm{pcm}$ and $\Lambda_{\text {eff }}=80.25522 \mu \mathrm{s}$, with $k_{\text {eff }}=1.000921$, hence $\tilde{\alpha}_{p} \simeq-77.59 \mathrm{~s}^{-1}$, in $\operatorname{good}$ agreement with the $\alpha$-eigenvalue calculation.

The computed value for $\alpha_{p}$ using the PARTISN code is $\alpha_{p}=-96.13 s^{-1}$ (Endo and Yamamoto, 2018), which is probably due to the differences between multigroup and continuous-energy treatment of neutron transport. For the deterministic calculations, $k_{\text {eff }}=0.99944$ and $\beta_{\text {eff }}=725 \mathrm{pcm}$ while generation time is $\Lambda_{\text {eff }}=$ $80.73 \mu s$ (Endo and Yamamoto, 2018). This small discrepancy in $k_{\text {eff }}$ (about 150 $\mathrm{pcm}$ ) is responsible for a much larger discrepancy in $\alpha_{p}$. Since the $\alpha_{p}$ values estimated by the Monte Carlo and deterministic calculations are quite different, it is reasonable to normalize the computed sensitivities by the ratio of the $\alpha_{p}$ values, as discussed above. We have thus rescaled the TRIPOLI- $4{ }^{\circledR}$ simulation results by the dimensionless factor $q=76.78 / 96.13$.

Table 8 displays the ten major contributors (in absolute value) to the energyintegrated sensitivity coefficients as computed by TRIPOLI- $4{ }^{\circledR}$ and PARTISN, and the relative difference between them. Overall, a very good agreement is observed between the deterministic and Monte Carlo calculations, with a relative 
difference below $2 \%$. The only exception is the sensitivity to the elastic scattering of ${ }^{27} \mathrm{Al}$, which displays a higher relative difference of 5\%. However, the Monte Carlo uncertainty for these quantities is about $4.6 \%$ (adjoint-weighted sensitivities for elastic and inelastic scattering show slow convergence also in standard IFP calculations for $k$-eigenvalue calculations (Kiedrowski and Brown, 2013)), meaning that these results are consistent. The calculations performed with PARTISN did not include the implicit sensitivity due to resonance self-shielding (Williams et al., 2001); however, numerical tests have shown that the impact of this contribution is negligible for the benchmark configuration examined here (Endo and Yamamoto, 2018).

\begin{tabular}{lccc}
\hline Isotope - reaction & $\mathrm{T} 4 \pm \sigma(\%)$ & PARTISN & T4 / PARTISN - \\
\hline${ }^{235} U \bar{v}_{p}$ & $-1.2940 \mathrm{e}+02 \pm 0.15$ & $-1.2783 \mathrm{e}+02$ & -0.0121 \\
${ }^{235} U \sigma_{f}$ & $-6.3748 \mathrm{e}+01 \pm 0.18$ & $-6.2916 \mathrm{e}+01$ & -0.0131 \\
${ }^{1} H \sigma_{n, \gamma}$ & $5.0845 \mathrm{e}+01 \pm 0.14$ & $5.0374 \mathrm{e}+01$ & -0.0093 \\
${ }^{1} H \sigma_{e l}$ & $-3.7759 \mathrm{e}+01 \pm 10.09$ & $-3.7021 \mathrm{e}+01$ & -0.0195 \\
${ }^{235} U \sigma_{n, \gamma}$ & $1.1691 \mathrm{e}+01 \pm 0.14$ & $1.1551 \mathrm{e}+01$ & -0.0120 \\
${ }^{16} O \sigma_{e l}$ & $-7.5922 \mathrm{e}+00 \pm 1.95$ & $-7.5062 \mathrm{e}+00$ & -0.0113 \\
${ }^{27} \mathrm{Al} \sigma_{e l}$ & $-1.6214 \mathrm{e}-01 \pm 4.60$ & $-1.5345 \mathrm{e}-01$ & -0.0536 \\
${ }^{234} U \sigma_{n, \gamma}$ & $1.5001 \mathrm{e}-01 \pm 0.16$ & $1.4855 \mathrm{e}-01$ & -0.0097 \\
${ }^{27} \mathrm{Al} \sigma_{n, \gamma}$ & $1.0448 \mathrm{e}-01 \pm 0.39$ & $1.0411 \mathrm{e}-01$ & -0.0036 \\
${ }^{238} U \sigma_{n, \gamma}$ & $8.9630 \mathrm{e}-02 \pm 0.27$ & $8.8836 \mathrm{e}-02$ & -0.0089 \\
\hline
\end{tabular}

Table 8: Ten major contributors (in absolute value) to the energy-integrated sensitivity coefficients of the time eigenvalue $\alpha$ using the ENDF/B-VII.1 library.

Figures 4 and 5 display the sensitivity profiles to the elastic scattering of ${ }^{1} \mathrm{H}$ (including $S_{\alpha, \beta}$ data) and the fission cross section of ${ }^{235} U$, respectively, using 26 
the 238-groups energy mesh defined in the SCALE software package (Rearden and Jessee, 2018). An example of (constrained) sensitivity to the prompt fission spectrum of ${ }^{235} U$ is shown in Fig. 6. A very good agreement is found between the TRIPOLI- $4{ }^{\circledR}$ and the PARTISN calculations in all energy groups.

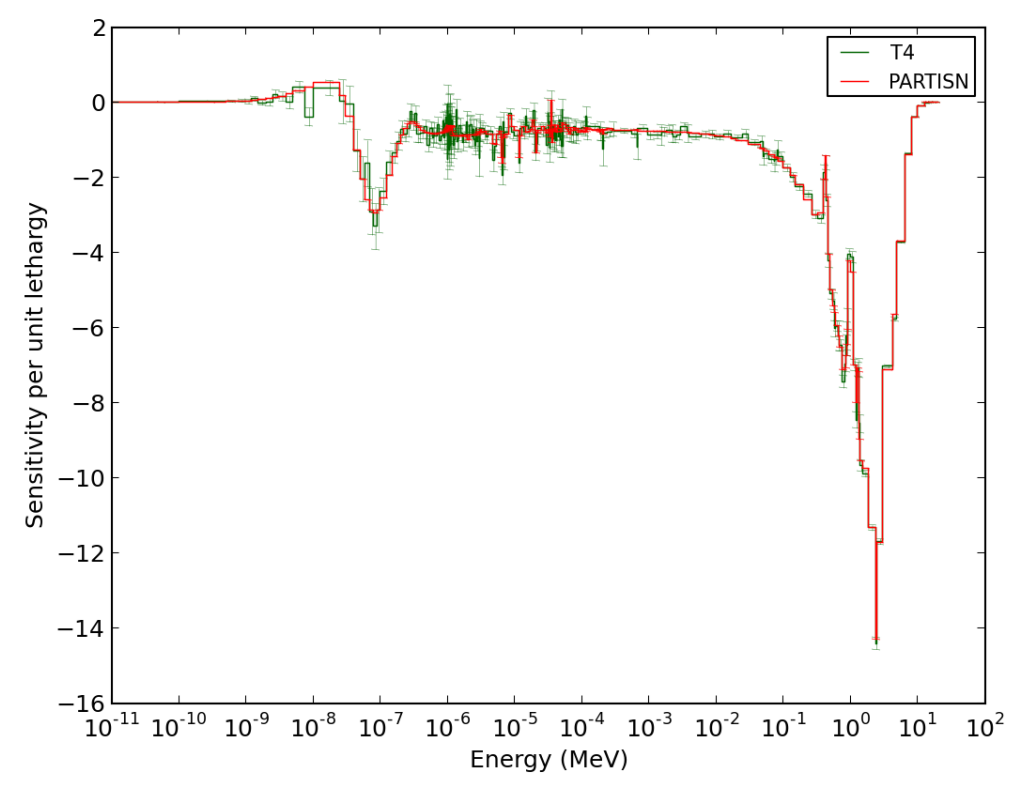

Figure 4: Sensitivity profile to the ${ }^{1} H$ elastic scattering cross section (including $S_{\alpha, \beta}$ data). Red: PARTISN calculation; green: TRIPOLI- $4^{\circledR}$ calculation.

In addition to comparison with the deterministic code PARTISN, we have also computed direct perturbations $\Delta \alpha$ of the time eigenvalue due to small variations of some nuclide concentrations. The $\alpha$ eigenvalues corresponding to a positive and negative perturbation have been computed, and a centered difference is calculated. This direct (exact) perturbation $\Delta \alpha$ has been then compared to a firstorder perturbation based on the sensitivity coefficients of the concentration, as obtained by the Generalized IFP method. Results are gathered in Tab. 9 and show a rather good agreement, which further corroborates the proposed algorithms for 


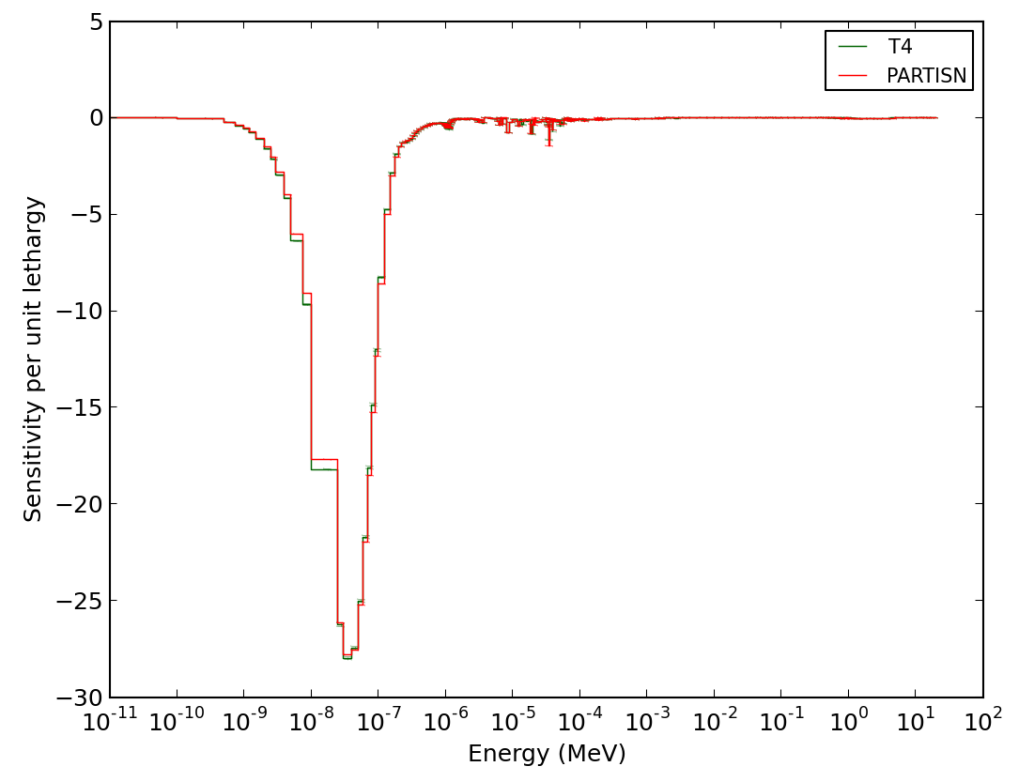

Figure 5: Sensitivity profile of the time eigenvalue $\alpha$ to the ${ }^{235} U$ fission cross section. Red: PARTISN calculation; green: TRIPOLI- $4^{\circledR}$ calculation.

perturbations and sensitivity analysis in continuous-energy transport problems.

\begin{tabular}{lcc}
\hline Isotope : perturbation & Exact $\Delta \alpha \pm \sigma(\%)$ & First-order $\Delta \alpha \pm \sigma(\%)$ \\
\hline${ }^{235} \mathrm{U}: 1 \%$ & $5.0739 \mathrm{e}+01 \pm 1.549$ & $5.0070 \mathrm{e}+01 \pm 0.239$ \\
${ }^{1} \mathrm{H}: 5 \%$ & $-6.4282 \mathrm{e}+01 \pm 1.257$ & $-6.2919 \mathrm{e}+01 \pm 4.396$ \\
${ }^{16} \mathrm{O}: 5 \%$ & $3.5080 \mathrm{e}+01 \pm 2.148$ & $3.5803 \mathrm{e}+01 \pm 1.995$ \\
\hline
\end{tabular}

Table 9: Comparison of direct exact perturbations and first-order perturbations for the time eigenvalue $\alpha$.

We conclude our analysis by assessing the impact of the nuclear data library on the obtained results. For this purpose, we have re-run our calculations using the JEFF-3.1.1 library (Santamarina et al., 2009). For the eigenvalue, we obtain $\alpha_{p}=-75.42 s^{-1}$. Energy-integrated sensitivity coefficients are compared in 


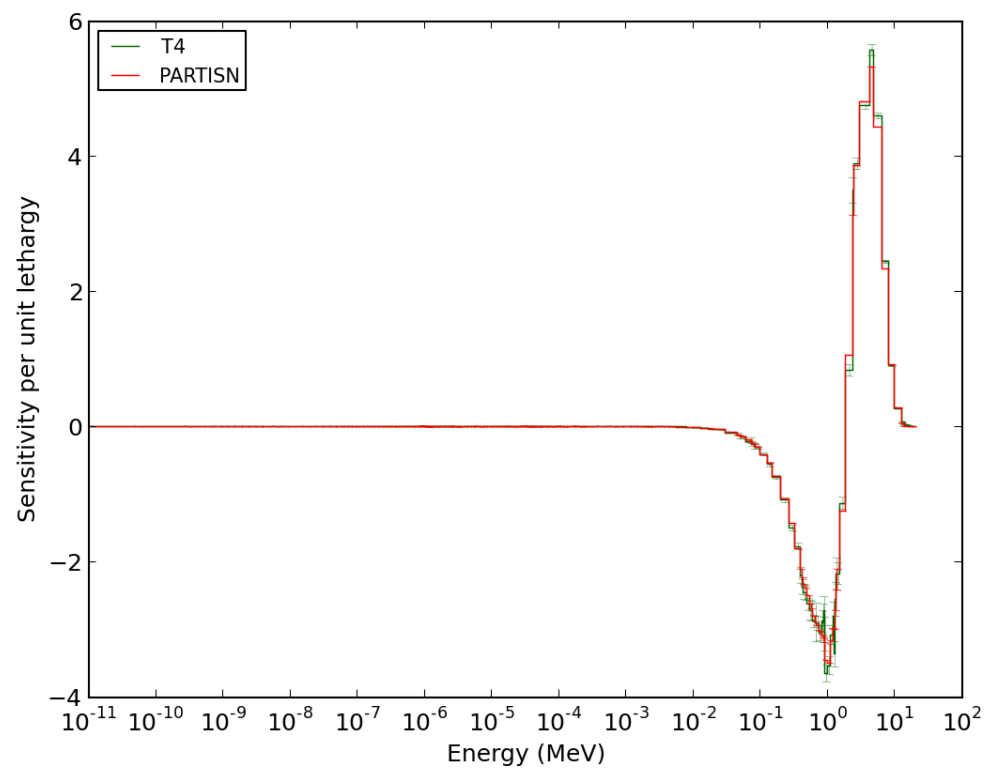

Figure 6: Sensitivity profile of the time eigenvalue $\alpha$ to the ${ }^{235} U$ prompt fission spectrum, with constrained coefficients (the integral sensitivity yields zero). Red: PARTISN calculation; green: TRIPOLI- $4{ }^{\circledR}$ calculation.

Tab. 10, where $\mathrm{R} 1$ is the relative difference between the results obtained by using the two nuclear data libraries (taking ENDF/B-VII.1 as the reference), and R2 is the relative difference divided by the combined standard deviations ${ }^{6}$. Simulation findings show that the discrepancies on the sensitivity coefficients induced by the choice of the nuclear data library are typically small for this benchmark configuration. An interesting exception is represented by the case of the sensitivity profile to ${ }^{16} \mathrm{O}$ radiative capture cross section (which however is not among the ten major contributors to sensitivities displayed in Tab. 10): for this cross section, a large discrepancy is found in the $\mathrm{MeV}$ region, as shown in Fig. 7. The observed effect on the sensitivity profile is actually due to the very

\footnotetext{
${ }^{6}$ Confidence intervals are consistent when $\mathrm{R} 2<3$.
} 
different shape of the cross sections in this energy region between JEFF-3.1.1 and ENDF/B-VII.1 libraries.

\begin{tabular}{lcccc}
\hline Isotope - reaction & ENDFB/VII. $1 \pm \sigma(\%)$ & $\mathrm{JEFF} 3.1 .1 \pm \sigma(\%)$ & $\mathrm{R} 1$ & $\mathrm{R} 2$ \\
\hline${ }^{235} U \bar{v}_{p}$ & $-1.6493 \mathrm{e}+02 \pm 0.15$ & $-1.6500 \mathrm{e}+02 \pm 0.17$ & 0.0004 & 0.17 \\
${ }^{235} U \sigma_{f}$ & $-8.1253 \mathrm{e}+01 \pm 0.18$ & $-8.1246 \mathrm{e}+01 \pm 0.19$ & -0.0001 & 0.04 \\
${ }^{1} H \sigma_{n, \gamma}$ & $6.4807 \mathrm{e}+01 \pm 0.14$ & $6.4840 \mathrm{e}+01 \pm 0.16$ & 0.0005 & 0.24 \\
${ }^{1} H \sigma_{e l}$ & $-4.8127 \mathrm{e}+01 \pm 10.09$ & $-4.9349 \mathrm{e}+01 \pm 9.18$ & 0.0254 & 0.19 \\
${ }^{235} U \sigma_{n, \gamma}$ & $1.4901 \mathrm{e}+01 \pm 0.14$ & $1.4910 \mathrm{e}+01 \pm 0.16$ & 0.0006 & 0.28 \\
${ }^{16} O \sigma_{e l}$ & $-9.6770 \mathrm{e}+00 \pm 1.95$ & $-9.7391 \mathrm{e}+00 \pm 2.00$ & 0.0064 & 0.23 \\
${ }^{27} \mathrm{Al} \sigma_{e l}$ & $-2.0666 \mathrm{e}-01 \pm 4.60$ & $-2.0572 \mathrm{e}-01 \pm 4.62$ & -0.0045 & 0.07 \\
${ }^{234} U \sigma_{n, \gamma}$ & $1.9120 \mathrm{e}-01 \pm 0.16$ & $1.8969 \mathrm{e}-01 \pm 0.18$ & -0.0079 & 3.32 \\
${ }^{27} \mathrm{Al} \sigma_{n, \gamma}$ & $1.3317 \mathrm{e}-01 \pm 0.39$ & $1.3225 \mathrm{e}-01 \pm 0.40$ & -0.0069 & 1.24 \\
${ }^{238} U \sigma_{n, \gamma}$ & $1.1424 \mathrm{e}-01 \pm 0.27$ & $1.1420 \mathrm{e}-01 \pm 0.27$ & -0.0003 & 0.09 \\
\hline
\end{tabular}

Table 10: Ten major contributors (in absolute value) to the energy-integrated sensitivity coefficients of the time eigenvalue $\alpha$ using the ENDF/B-VII.1 and JEFF-3.1.1 nuclear data libraries, with the former as the reference. 


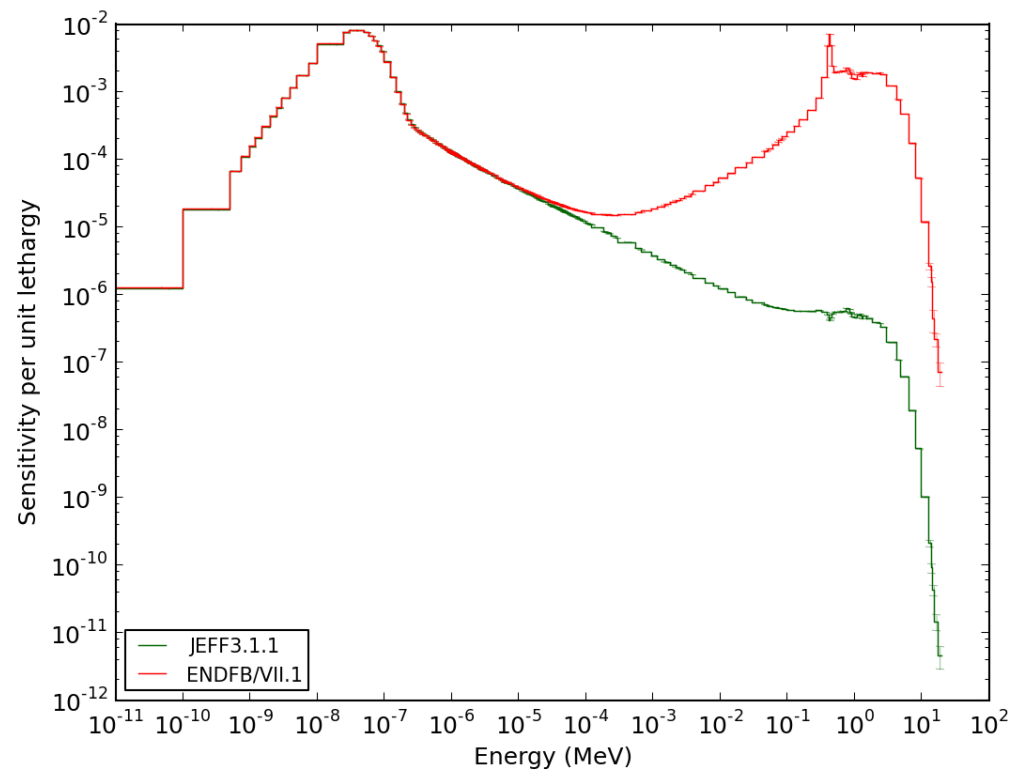

Figure 7: Sensitivity profiles of the time eigenvalue $\alpha$ to ${ }^{16} O$ radiative capture cross section using the ENDF/B-VII.1 (red curve) and JEFF-3.1.1 (green curve) nuclear data libraries. Although the applied sensitivity methodology is identical for both cases, a large discrepancy is found in the $\mathrm{MeV}$ region, due to the very different shape of the cross sections in this energy range between JEFF-3.1.1 and ENDF/B-VII.1 libraries.

\section{Conclusions}

A new method for computing time-eigenvalue sensitivity coefficients and perturbations has been successfully developed and implemented within the frame of a $\alpha-k$ iteration scheme. This method relies on the Generalized IFP method that we have recently introduced for continuous-energy Monte Carlo transport codes and shares some features with the standard IFP algorithm for $k$-eigenvalue calculations. Specific tallies designed to estimate the terms appearing in the formulas for perturbations and sensitivities have been devised. The proposed strategy has been first tested for simple configurations where analytic expressions exist for $\alpha$ eigenvalues and eigenfunctions, in continuous-energy and multi-group 
transport, so that reference solutions can be computed for the first-order $\Delta \alpha$ perturbations and the associated sensitivities. The method has been implemented in the development version of TRIPOLI- $4{ }^{\circledR}$ and compared to the simulation findings obtained from the deterministic solver PARTISN for the HEU-SOL-THERM-012 benchmark. In all tested cases, the proposed algorithm has shown satisfactory results in terms of accuracy and robustness.

Several independent implementations of perturbations and sensitivities for time eigenvalues have been recently published, encompassing both deterministic and Monte Carlo solvers (Endo and Yamamoto, 2018; Favorite, 2018; Yamamoto and Sakamoto, 2019; Burke et al., 2019; Jinaphanh and Zoia, 2019): it might be then interesting to compare the distinct merits and drawbacks of each method on some benchmark configurations.

\section{Acknowledgements}

The authors express their gratitude to Dr. Tomohiro Endo (Graduate School of Engineering, Nagoya University) for providing digitalized data corresponding to the figures presented in his work (Endo and Yamamoto, 2018). TRIPOLI-4 ${ }^{\circledR}$ is a registered trademark of CEA. The authors wish to thank Électricité de France (EDF) for partial financial support.

\section{References}

R. E. Alcouffe et al. PARTISN: A Time-Dependent, Parallel Neutral Particle Transport Code System. Technical Report LA-UR-17-29704 (Revised Oct. 2018), Los Alamos National Laboratory, 2018.

M. Aufiero, A. Bidaud, M. Hursin, J. Leppnen, G. Palmiotti, S. Pelloni, and P. Rubiolo. A collision history-based approach to sensitivity/perturbation calculations in the continuous energy Monte Carlo code SERPENT. Annals of Nuclear Energy, 85:245 - 258, 2015. 
G. Bell and S. Glasstone. Nuclear Reactor Theory. Van Nordstrand Reinhold Company, 1970.

D. Brockway, P. Soran, and P. Whalen. Monte Carlo alpha calculations. Technical Report LA-UR-85-1224, Los Alamos National Laboratory, 1985.

E. Brun, F. Damian, C. Diop, E. Dumonteil, F. Hugot, C. Jouanne, Y. Lee, F. Malvagi, A. Mazzolo, O. Petit, J. Trama, T. Visonneau, and A. Zoia. TRIPOLI- $4^{\circledR}$, CEA, EDF and AREVA reference Monte Carlo code. Annals of Nuclear Energy, 82:151 - 160, 2015.

T. P. Burke, C. J. Josey, and B. C. Kiedrowski. personal communication, 2019.

Y. Cao and J. C. Lee. Spatial corrections for pulsed-neutron reactivity measurements. Nuclear Science and Engineering, 165(3):270-282, 2010.

M. Chadwick et al. Endf/b-vii.1 nuclear data for science and technology: Cross sections, covariances, fission product yields and decay data. Nuclear Data Sheets, 112(12):2887 - 2996, 2011.

S. H. Choi, H. J. Shim, and C. H. Kim. Development of Generalized Perturbation Theory Algorithms for Monte Carlo Eigenvalue Calculations. Nuclear Science and Engineering, 189 (2):171-187, 2018.

D. Cullen. Static and dynamic criticality: are they different? Technical Report UCRL-TR-201506, University of California Radiation Laboratory, 2003.

J. Duderstadt and W. Martin. Transport theory. J. Wiley and sons, New York, 1979.

T. Endo and A. Yamamoto. Sensitivity analysis of prompt neutron decay constant using perturbation theory. Journal of Nuclear Science and Technology, 55:1245-1254, 2018.

J. A. Favorite. SENSMG: First-Order Sensitivities of Neutron Reaction Rates, Reaction-Rate Ratios, Leakage, keff, and $\alpha$ Using PARTISN. Nuclear Science and Engineering, 192(1): 80-114, 2018.

T. Hill. Efficient methods for time absorption (alpha) eigenvalue calculations. Technical Report LA-9602-MS (UC-32), Los Alamos National Laboratory, 1983.

J. Hoogenboom. Numerical calculation of the delayed-alpha eigenvalue using a standard criticality code. In Physor 2002, Seoul, Korea, 2002.

ICSBEP. International Handbook of Evaluated Criticality Safety Benchmark Experiments. NEA/NSC/DOC(95)/03, OECD NEA, Paris, France, 2018.

A. Jinaphanh and A. Zoia. Perturbation and sensitivity of the time eigenvalues by a Generalized Iterated Fission Probability method. Manuscript submitted for publication at M\&C 2019, 2019. 
A. Jinaphanh, N. Leclaire, and B. Cochet. Continuous-Energy Sensitivity Coefficients in the MORET Code. Nuclear Science and Engineering, 184(1):53-68, 2016.

C. J. Josey. General Improvements to the MCNP Alpha-Eigenvalue Solver. Technical Report LA-UR-18-22738, Los Alamos National Laboratory, 2018.

B. C. Kiedrowski. Analytic, Infinite-Medium Solutions for Point Reactor Kinetics Parameters and Reactivity Perturbations. Technical Report LA-UR-10-01803, Los Alamos National Laboratory, 2010.

B. C. Kiedrowski. Review of Early 21st-Century Monte Carlo Perturbation and Sensitivity Techniques for k-Eigenvalue Radiation Transport Calculations. Nuclear Science and Engineering, 185(3):426-444, 2017.

B. C. Kiedrowski and F. B. Brown. Adjoint-Based k-Eigenvalue Sensitivity Coefficients to Nuclear Data Using Continuous-Energy Monte Carlo. Nuclear Science and Engineering, 174 (3):227-244, 2013.

B. C. Kiedrowski, F. B. Brown, and P. P. H. Wilson. Adjoint-Weighted Tallies for k-Eigenvalue Calculations with Continuous-Energy Monte Carlo. Nuclear Science and Engineering, 168(3): 226-241, 2011.

D. Mancusi and A. Zoia. Chaos in eigenvalue search methods. Annals of Nuclear Energy, 112: $354-363,2018$.

Y. Nauchi. Attempt to estimate reactor period by natural mode eigenvalue calculation. In $S N A+M C$ 2013, Paris, France, 2013.

Y. Nauchi and T. Kameyama. Development of Calculation Technique for Iterated Fission Probability and Reactor Kinetic Parameters Using Continuous-Energy Monte Carlo Method. Journal of Nuclear Science and Technology, 47:977-990, 2010.

Y. Nauchi, A. Jinaphanh, and A. Zoia. Verification of adjoint functions of natural mode equation by Generalized Iterated Fission Probability method and by analog Monte Carlo. Manuscript submitted for publication at M\&C 2019, 2019.

C. M. Perfetti, B. T. Rearden, and W. R. Martin. SCALE Continuous-Energy Eigenvalue Sensitivity Coefficient Calculations. Nuclear Science and Engineering, 182(3):332-353, 2016.

W. Pfeiffer, J. Brown, and A. Marshall. Fort St. Vrain startup test A-3: Pulsed-Neutron experiments. Technical Report GA-A13079, General Atomic, 1974.

Y. Qiu, M. Aufiero, K. Wang, and M. Fratoni. Development of sensitivity analysis capabilities of 
generalized responses to nuclear data in Monte Carlo code RMC. Annals of Nuclear Energy, 97:142-152, 2016.

B. T. Rearden and M. Jessee. SCALE Code System Version 6.2.3. Technical Report ORNL/TM2005/39, Oak Ridge National Laboratory, 2018.

A. Santamarina et al. The JEFF-3.1.1 Nuclear Data Library. Technical Report JEFF Report 22, OECD-NEA Data Bank, 2009.

N. Terranova, D. Mancusi, and A. Zoia. Generalized Iterated Fission Probability for Monte Carlo eigenvalue calculations. Annals of Nuclear Energy, 108:57-66, 2017.

N. Terranova, D. Mancusi, and A. Zoia. New perturbation and sensitivity capabilities in TRIPOLI$4^{\circledR}$. Annals of Nuclear Energy, 121:335-349, 2018.

G. Truchet, P. Leconte, A. Santamarina, E. Brun, F. Damian, and A. Zoia. Computing adjointweighted kinetics parameters in TRIPOLI- ${ }^{\circledR}$. Annals of Nuclear Energy, 85:17-26, 2015.

M. L. Williams, B. L. Broadhead, and C. V. Parks. Eigenvalue sensitivity theory for resonanceshielded cross sections. Nuclear Science and Engineering, 138:177-191, 2001.

T. Yamamoto and H. Sakamoto. A Monte Carlo technique for sensitivity analysis of alphaeigenvalue with the differential operator sampling method. Annals of Nuclear Energy, 127: 178-187, 2019.

A. Zoia, E. Brun, and F. Malvagi. Alpha eigenvalue calculations with TRIPOLI- $4{ }^{\circledR}$. Annals of Nuclear Energy, 63:276 - 284, 2014.

A. Zoia, E. Brun, F. Damian, and F. Malvagi. Monte Carlo methods for reactor period calculations. Annals of Nuclear Energy, 75:627 - 634, 2015. 